\title{
Performance robustness and stiffness analysis on a machine tool servo design
}

\author{
Jia-Yush Yen $^{\mathrm{a}, *}$, Hui-Man Chang ${ }^{\mathrm{b}}$ \\ a Department of Mechanical Engineering, National Taiwan University, Taipei 10617, Taiwan \\ b ASUSTek Computer Inc., Taipei, Taiwan
}

Received 16 April 2003; received in revised form 16 October 2003; accepted 22 October 2003

\begin{abstract}
The machine tool servo design is very different from the traditional high performance servo systems. Traditional servo design depends heavily on a precise system model so that frequency domain or time domain compensation techniques can be applied, and many synthesis tools such as $\mathrm{LQG}, \mathrm{H}_{\infty}$, or LMI, ... are available based on this concept. While these synthesis tools are becoming very useful in many situations, they are not used in the machine tool servo design. Most high performance machine tool systems still rely on the more primitive PID or PDF controllers in conjunction with complicated friction and temperature compensation algorithms. This paper investigates the different approaches based on a house-designed servo control board. With the ability to change the servo control algorithms on a test machine tool, the performance robustness and servo stiffness were tested both numerically and experimentally. The numerical tests conducted with PID, PDF, and LQG controllers showed that the PID controller is the easiest to achieve good performance, but the PDF controller contains the best performance robust margin. Experimental results also indicated that the PDF controller exhibits far superior robustness properties over the other two controllers.
\end{abstract}

(C) 2003 Elsevier Ltd. All rights reserved.

Keywords: Machine tool servo; PDF control; Stiffness robust analysis

\section{Introduction}

The CNC machine tool servo uses a very different design philosophy to formulate its control algorithm. The machine tool servo design does not stress a precise system model. Instead, the servo system tries to achieve very high performance with very good robustness properties. It is quite understandable in the machine tool servo design that the precise model is not emphasized. Usually, machine dynamics contributes relatively little effect during cutting. The system experiences mainly the cutting force and the material removing action. It would not be practical for the servo system design to be based on any particular cutting process, and a robust universally high performance system is desirable.

\footnotetext{
* Corresponding author.

E-mail address: jyen@ccms.ntu.edu.tw (J.-Y. Yen).
}

Looking more closely into the design, one notices that even though many modern control design techniques are now available, most machine tool servo designs are still based on the well-known PDF control architecture. More delicate processes are then added to eliminate the effect of backlash, axis spikes, friction, etc. Previous research has discussed machine servo problems in some specific domains like stiction, axes spikes, etc. [1-7]. Some of the results have tried to add machine intelligence to improve servo performance [8-18]. Very few results have directly addressed the problem of the most suitable underlying control algorithm.

This paper examines the behavior of the most commonly used controllers, such as the PID controller, the PDF controller $[19,20]$ and the model based compensators [21,22], and offers a reasonable comparison based on the machine tool servo considerations. Because the tests are conducted on a programmable controller with a commercial EDM machine, the study should be practical and should offer better insight to the interested 


\begin{tabular}{|ll|}
\hline Nomenclature \\
$G \quad$ Plant transfer function, $G(s)$ \\
$A$ & System matrix in the state space representation \\
$B$ & System input matrix in the state space representation \\
$C$ & System output matrix in the state space representation \\
$K$ & Proportional gain in the PID and PDF control \\
$K_{d}$ & Pseudo derivative gain in the PDF control \\
$K_{\mathrm{M}}$ & State feedback gain in the model based control \\
$L_{\mathrm{M}}$ & State estimator gain in the model based control \\
$\Delta_{K}$ & Variation in PID and PDF proportional gain \\
$\Delta_{K d}$ & Variation in the PDF pseudo derivative gain \\
$\Delta_{L}$ & Variation in the state feedback gain \\
$\Delta_{K}$ & Variation in the state estimator gain \\
$S$ & Laplace operator \\
$y$ & System output (work table position) \\
$r$ & Reference input (position command) \\
$\tilde{u}$ & Manipulated input term in the LFT representation \\
$\tilde{z}$ & Feedback signal term in the LFT representation \\
$Q_{\mathrm{ij}}$ & The transfer function from the $j$ th input to the $i$ th output in the LFT representation \\
$T$ & $T(s)=\left(s I-A-B K_{\mathrm{M}}+L_{\mathrm{M}} C\right)^{-1}$ \\
$F$ & Force disturbance input \\
\hline
\end{tabular}

reader. Both the performance robustness margins and the stiffness robustness against controller parameter variations were analyzed and experimentally tested. Both results agree with each other, and the fact that PDF controllers exhibit the best robustness properties may have been the reason that it has been the most widely used control architecture in the commercial machine tool controllers.

\section{System description}

The experiment is based on a PC-based CNC controller implemented on a small-hole EDM machine (Fig. 1). The CNC controller is the result of a project between the NTU Precision System Control Laboratory with the Chanceux Co., Taiwan.

The CNC controller is composed of an industrial PC with a house-designed motion card. The IPC runs Windows CE for user interface and task coordination. It allows trajectory programming, speed specification, s-curve motion, etc. The motion card is based on a TMS320F243 microcontroller [23,24,25] with an FPGA to decode the optical encoder and to communicate with the IPC. The motion card is equipped with four channel 12-bit $\mathrm{D} / \mathrm{A}$ for multi-axis servo control. The encoder on the EDM machine has a resolution of $5 \mu \mathrm{m}$, with three $180 \mathrm{~W}$ DC servo motor to drive the different axes. The tachometer sensitivity on the servomotor is $7 \mathrm{~V} / \mathrm{Krmp}$.

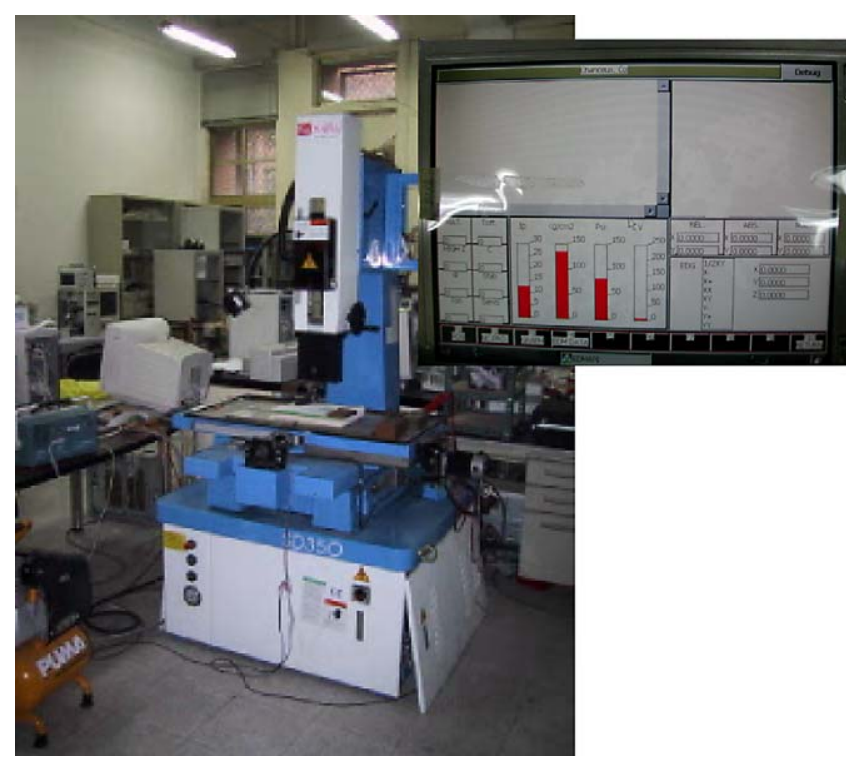

Fig. 1. The EDM machine and the CNC controller interface.

The controller set-up basically allows for not only the change of the control parameters, but also allows for the reprogramming of the controller structure. Therefore different servo control algorithms can be implemented on the test machine. This paper examined the popular PID controller, the PDF controller, and the more advanced model-based compensator (MBC) derived from the LQG. 


\section{Robustness analysis}

The system identification result for the y-axis motion table on the EDM machine is shown in Fig. 2. The servo controller is then designed based on the identification result.

This paper examined the popular PID controller, the PDF controller, and the more advanced model-based compensator (MBC) derived from the LQG. According to the machine set-up for parameter tuning, the system uncertainties due to controller parameter can be represented as follows.

\subsection{PID controller}

The PID controller takes the output position error from the worktable and computes the control effort. It is intuitive to treat the controller as a single block. Thus it is reasonable to treat the controller variation as a multiplicative uncertainty located at the output side of the controller.

Let $G$ denote the plant transfer function obtained from Fig. 2, and let $\mathrm{K}$ denote the controller, and $\Delta_{\mathrm{K}}$ denote the multiplicative uncertainty. The system block diagram with uncertainty can be rearranged as Fig. 3, and the system transfer function can be expressed as

$y(s)=G \tilde{z}(s)+G \tilde{u}(s)$, or

$y(s)=\left[G-G(I+K G)^{-1} K G\right] \tilde{z}(s)$

$$
+G(I+K G)^{-1} K r(s)
$$

$y(s)=G(I+K G)^{-1} \tilde{z}(s)+G K(I+G K)^{-1} r(s)$.

The manipulated input, $\tilde{u}$, can be expressed as

$$
\begin{aligned}
& \tilde{u}(s)=K r(s)-K G \tilde{u}(s)-K G \tilde{z}(s), \text { or } \\
& \tilde{u}(s)=(I+K G)^{-1} K r(s)-(I+K G)^{-1} K G \tilde{z}(s) .
\end{aligned}
$$

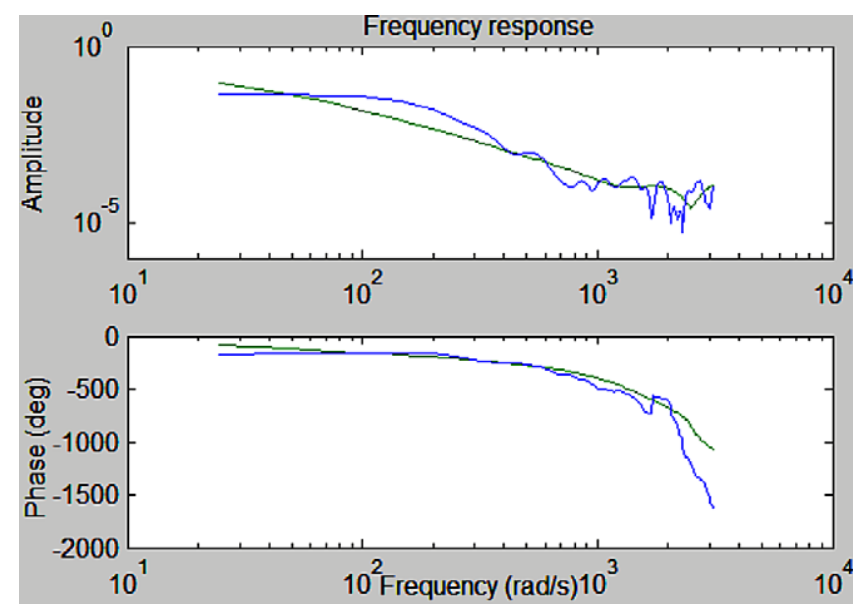

Fig. 2. The machine tool plant system identification result.

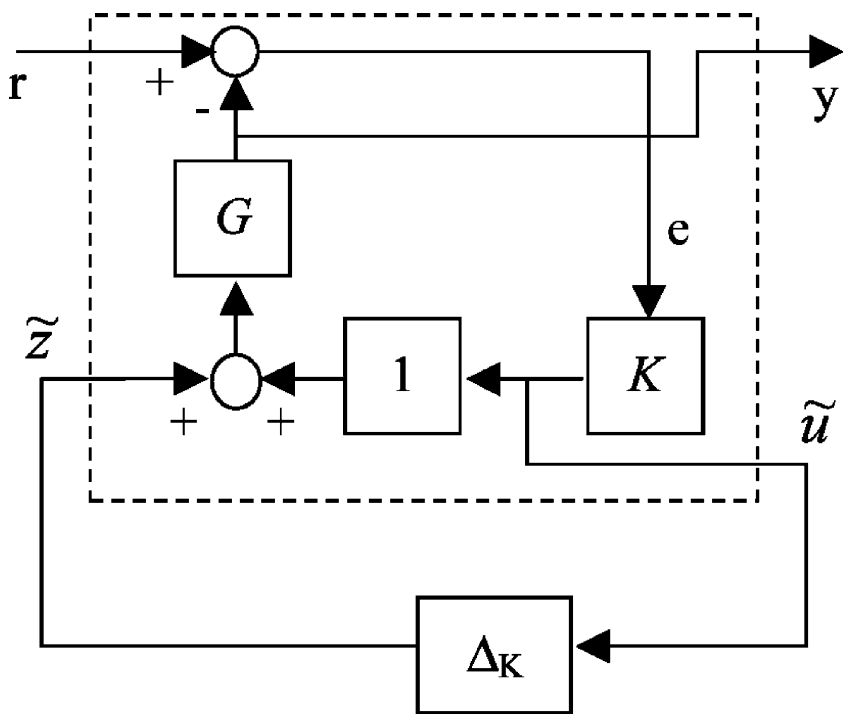

Fig. 3. The LFT structure for PID control system.

The system can now be put into the linear fractional transformation (LFT) for analysis. The LFT representation for the PID control system is, thus,

$\left[\begin{array}{l}\tilde{u} \\ y\end{array}\right]=\left[\begin{array}{ll}Q_{11} & Q_{12} \\ Q_{21} & Q_{22}\end{array}\right]\left[\begin{array}{l}\tilde{z} \\ r\end{array}\right]$

where from (5),

$Q_{11}=-(I+K G)^{-1} K G$

$Q_{12}=K(I+G K)^{-1}$, and from $(3)$

$Q_{21}=(I+G K)^{-1} G$

$Q_{22}=(I+G K)^{-1} G K$.

\subsection{PDF control}

The PDF controller uses both the output position and the positioning error for its inputs; therefore, the controller variations for the proportional gain and the differential gain are treated separately. The block diagram for the system can now be rearranged as in Fig. 4.

The PDF control system can be described by

$$
\begin{aligned}
& \tilde{u}_{1}=K\left[r-G\left(-\tilde{u}_{1}+\tilde{z}_{1}+\tilde{u}_{2}+\tilde{z}_{2}\right)\right] \\
& \tilde{u}_{2}=K_{f d} S G\left(-\tilde{u}_{1}+\tilde{z}_{2}+\tilde{u}_{2}+\tilde{z}_{2}\right) \\
& y=G\left(-\tilde{u}_{1}+\tilde{z}_{1}+\tilde{u}_{2}+\tilde{z}_{2}\right) .
\end{aligned}
$$

Eqs. (11-13) can be solved for $\tilde{u}_{1}, \tilde{u}_{2}$ and $y$ to obtain

$$
\begin{aligned}
\tilde{u}_{1}= & -K G\left(I+K G+K_{f d} S G\right)^{-1} \tilde{z}_{1}+K G(I+K G \\
& \left.+K_{f d} S G\right)^{-1} \tilde{z}_{2}+\left(I+K_{f d} S G\right)(I+K G \\
& \left.+K_{f d} S G\right)^{-1} K r
\end{aligned}
$$




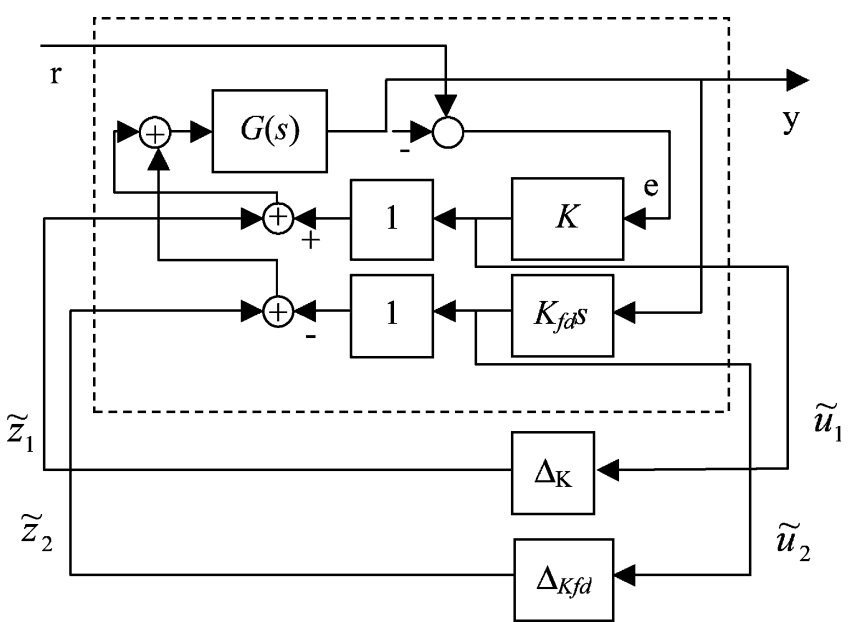

Fig. 4. The LFT structure for the PDF control system.

$$
\begin{aligned}
\tilde{u}_{2}= & K_{f d} s G\left(I+K G+K_{f d} S G\right)^{-1} \tilde{z}_{1}-K_{f d} S G(I+K G \\
& \left.+K_{f d} s G\right)^{-1} \tilde{z}_{2}+K_{f d} S G\left(I+K G+K_{f d} S G\right)^{-1} K r \\
y= & G\left(I+K G+K_{f d} S G\right)^{-1} \tilde{z}_{1}-G(I+K G \\
& \left.+K_{f d} s G\right)^{-1} \tilde{z}_{2}+G\left(I+K G+K_{f d} S G\right)^{-1} K r
\end{aligned}
$$

Eqs. (14-16) can again be rearranged into LFT representation to give

$\left[\begin{array}{l}\tilde{u}_{1} \\ \tilde{u}_{2} \\ y\end{array}\right]=\left[\begin{array}{lll}Q_{11} & Q_{12} & Q_{13} \\ Q_{21} & Q_{22} & Q_{23} \\ Q_{31} & Q_{32} & Q_{33}\end{array}\right]\left[\begin{array}{l}\tilde{z}_{1} \\ \tilde{z}_{2} \\ r\end{array}\right]$

where

$$
\begin{aligned}
& Q_{11}=-K G\left(I+K G+K_{f d} s G\right)^{-1} \\
& Q_{12}=K G\left(I+K G+K_{f d} s G\right)^{-1} \\
& Q_{13}=\left(I+K_{f d} s G\right)\left(I+K G+K_{f d} s G\right)^{-1} K
\end{aligned}
$$

$$
\begin{aligned}
& Q_{21}=K_{d} s G\left(I+K G+K_{f d} s G\right)^{-1} \\
& Q_{22}=-K_{d} s G\left(I+K G+K_{f d} s G\right)^{-1} \\
& Q_{23}=K_{d} s G\left(I+K G+K_{f d} s G\right)^{-1} K \\
& Q_{31}=G\left(I+K G+K_{f d} s G\right)^{-1} \\
& Q_{32}=-G\left(I+K G+K_{f d} s G\right)^{-1} \\
& Q_{33}=G\left(I+K G+K_{f d} s G\right)^{-1} K .
\end{aligned}
$$

\subsection{Model-based compensator}

The basic LFT structure for the MBC system is treated as in Fig. 5.

The MBC control system derived from the LQG design procedure requires separate computation of a state feedback gain and an estimator gain. It is thus reasonable to separately treat the variations for the state feedback gain, $K_{\mathrm{M}}$, and the observer gain, $L_{\mathrm{M}}$. Let $\Delta_{\mathrm{L}}$ stands for the variation in the estimator gain $L_{\mathrm{M}}$, and $\Delta_{\mathrm{K}}$ stands for the variation in the state feedback gain $K_{\mathrm{M}}$, then similar to the PID and the PDF cases, the LFT representation can then be written as:

$$
\begin{aligned}
& \tilde{u}_{1}=L_{M}\left[r-y-C(s I-A)^{-1}\left[B\left(\tilde{z}_{2}+\tilde{u}_{2}\right)+\left(\tilde{z}_{1}+\tilde{u}_{1}\right)\right]\right] \\
& \tilde{u}_{2}=K_{M}\left[(s I-A)^{-1}\left[B\left(\tilde{z}_{2}+\tilde{u}_{2}\right)+\left(\tilde{z}_{1}+\tilde{u}_{1}\right)\right]\right] \\
& y=G\left[\tilde{z}_{2}+\tilde{u}_{2}\right] .
\end{aligned}
$$

One can solve the above equations for $\tilde{u}_{1}, \tilde{u}_{2}$, and $y$, and let $T=\left(s I-A-B K_{M}+L_{M} C\right)^{-1}$ to obtain

$$
\left[\begin{array}{l}
\tilde{u}_{1} \\
\tilde{u}_{2} \\
y
\end{array}\right]=\left[\begin{array}{lll}
Q_{11} & Q_{12} & Q_{13} \\
Q_{21} & Q_{22} & Q_{23} \\
Q_{31} & Q_{32} & Q_{33}
\end{array}\right]\left[\begin{array}{l}
\tilde{z}_{1} \\
\tilde{z}_{2} \\
r
\end{array}\right]
$$

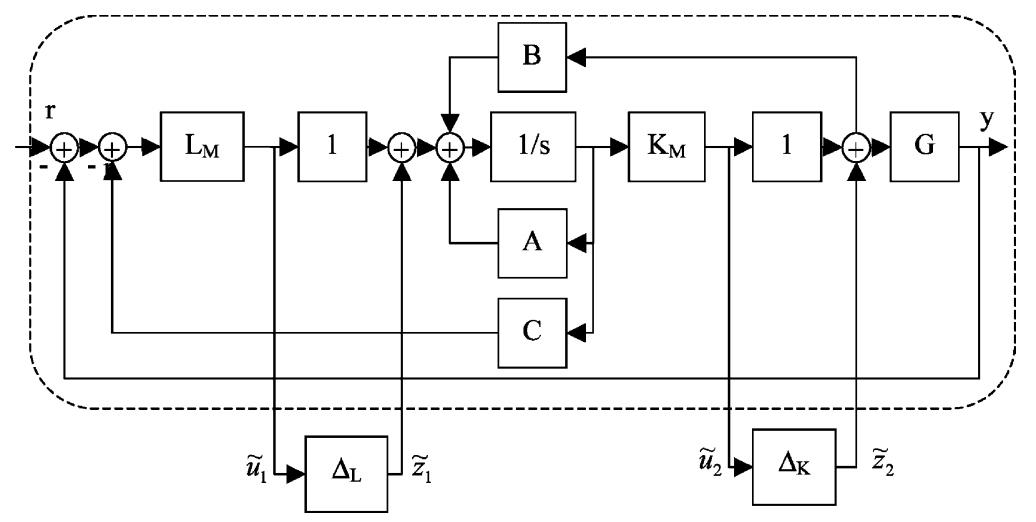

Fig. 5. The LFT structure for MBC control system. 
Then

$$
\begin{aligned}
& Q_{11}=\left(L_{M} C T L_{M}-L_{M}\right) Q_{31}-L_{M} C T \\
& Q_{12}=\left(L_{M} C T L_{M}-L_{M}\right) Q_{32}-L_{M} C T B \\
& Q_{13}=\left(L_{M} C T-I\right) L_{M}\left(I+Q_{33}\right) \\
& Q_{21}=K_{M} T\left(I-L_{M} Q_{31}\right) \\
& Q_{22}=K_{M} T B-K_{M} T L_{M} Q_{32} \\
& Q_{23}=K_{M} T L_{M}-K_{M} Y L_{M} Q_{33} \\
& Q_{31}=\left(I+G K_{M} T L_{M}\right)^{-1} G K_{M} T \\
& Q_{32}=\left(I+G K_{M} T L_{M}\right)^{-1}\left(G+G K_{M} T B\right) \\
& Q_{33}=\left(I+G K_{M} T L_{M}\right)^{-1} G K_{M} T L_{M} .
\end{aligned}
$$

With the LFT for the different types of control algorithm, we can perturb the parameters with $20,40,60,80$ and $100 \%$ of their nominal values. The robust performance margin can be calculated with MATLAB. If the computed robust performance margin is greater than 1 , the system performance is robust for the specified control gain variations.

\subsection{Performance robustness}

Fig. 6 shows the robust performance margin for the PID and PDF control system. One observes better performance robustness with the PDF controller. The PID control maintains performance robustness until around $35 \%$ of gain variation. The PDF controller, on the other hand, maintained performance robustness until close to $50 \%$ gain variation.

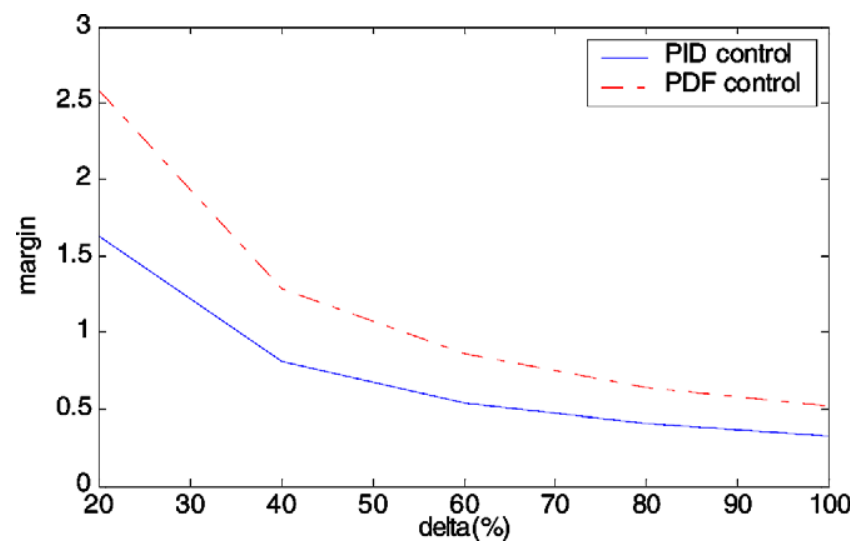

Fig. 6. Robust performance margin for PID and PDF control systems.

\subsection{Servo stiffness}

Servo stiffness is the most important factor for machine tool servo consideration. There are some discussions in the literature concerning the cutting stiffness. In particular Alter and Tsao talked about the definition of dynamic servo stiffness, which may help the purpose of this analysis [2]. The disturbance force model for the analysis is illustrated in Fig. 7. The servo stiffness is defined as the change in output displacement $y$ with respect to per unit disturbance cutting force, $F$.

The closed-loop transfer function from the disturbance cutting force to the output displacement can then be derived as:

PID controller

$\frac{G}{1+G\left(K_{p}+K_{i} / s+K d_{s}\right)}$

PDF controller

$$
\frac{G}{1+G\left(K_{p}+K_{i} / s+K_{d} s\right)+G K_{f d} s}
$$

Model-based compensator:

$$
\left[1+G K_{M}\left(s-A-B K_{M}+L_{M} C\right)^{-1} L_{M}\right]^{-1} G \text {. }
$$

From Eqs. (37)-(39), one can calculate the dynamic servo stiffness for the different control algorithms. Basically, the servo stiffnesses achieved with the three controllers are different. The controller parameters are for the PID controllers $K_{p}=80, K_{i}=1$, and $K_{d}=96$ (The numbers are implemented with an integer DSP, therefore, they have no dimensions. The numbers are for the experimental motion control card and the test EDM machine.) The PDF controller parameters are $K_{p}=80, K_{i}=8, K_{d}=80$, and $K_{f d}=10$, and the parameters for the $\mathrm{MBC}$ are $K_{M}=\left[\begin{array}{ll}0.7549 & 0.0039\end{array}\right]$ and $L_{M}=\left[\begin{array}{l}203524 \\ 132542\end{array}\right]$. With these tuned results, it is noted that the robustness properties against parameter variations are also different. We calculated the servo stiffness variation against the percentage gain variation for the different controllers.

Table 1 lists the changes in the servo stiffness in (units $/ \mathrm{m}$ ) against the controller parameter variations (\%). Table 1 shows that, somewhat to our surprise, the primitive PID controller achieves the best servo stiffness of 443.3 units $/ \mathrm{m}$. The PDF controller achieves

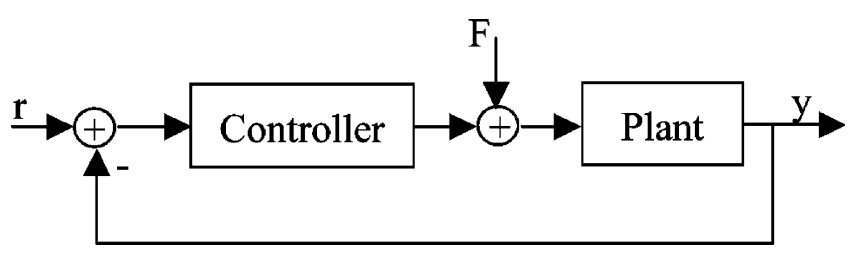

Fig. 7. Cutting force disturbance. 
Table 1

Servo stiffness for different controllers

\begin{tabular}{lllrrrr}
\hline & \multicolumn{1}{l}{0} & \multicolumn{1}{c}{$200 \%$} & \multicolumn{1}{c}{$400 \%$} & \multicolumn{1}{c}{$600 \%$} & \multicolumn{1}{c}{$800 \%$} & \multicolumn{1}{c}{$1000 \%$} \\
\hline PID & 443.2862 & 277.3089 & 277.3089 & 277.3089 & 277.2360 & 277.2360 \\
PDF & 138.9459 & 138.9459 & 138.9459 & 138.9459 & 138.9459 & 138.9459 \\
Model-based & 83.8630 & 42.8423 & 42.8423 & 14.2808 & 10.7106 & 8.5954 \\
\hline
\end{tabular}

a moderate 138.9 units $/ \mathrm{m}$. The more advanced modelbased compensator, on the other hand, achieves only 83.9 units $/ \mathrm{m}$ of stiffness. The variations against parameter changes are also very different among the controllers. The PDF controller maintained almost constant servo stiffness of 138.9 units/m even with a 10 times change in the controller gain. The PID controller has the achieved stiffness reduced in half (from 443.3 to 277.2 units $/ \mathrm{m}$ ) within 10 times gain variation. The MBC showed the worst 10-fold change (83.9 to 8.60 units $/ \mathrm{m}$ ) in its stiffness. Again, we are not sure if some kind of loop recovery technique can help in the stiffness robustness. Otherwise, PDF controller offers the best stiffness robustness property against controller gain variation.

\section{Experimental results}

These margins are illustrated by actually implementing them on the test machine. The experimental results show the response of the machine making a $1.25 \mathrm{~mm}$ step move. Fig. 8 shows the experimental result of the effect of gain variation on the system performance with PID controller. One can easily see that the rise time for the system sustained $100 \%$ gain variation and maintained stability; however, vibration starts appearing when the variation exceeds $40 \%$. Fig. 9 shows the effect of gain variation on the PDF controller. System stability is still maintained throughout. The vibration does not appear until there is $60 \%$ gain variation. The situations for the model-based compensator are shown in Fig. 10. We did not show the robustness margin curve for the model-based compensator because it is extremely sensitive to parameter variation. The experimental results agree with the calculation. The machine starts vibrating seriously with only very small changes in the state feedback gain or the observer gain. It is clear that some form of loop recovery is necessary in this case. Basically, all three situations closely agree with the calculation. Both the numerical results and the experimental results showed that the PDF controller maintains performance robustness against up to $50 \%$ of gain perturbation; while the PID controller only sustained performance robustness up to $35 \%$.

As for the servo stiffness, the experiments are carried out with a force pushing on the worktable of the machine. Figs. 11-13 show the resulted closed-loop dis- turbance force responses. The solid line in the figures represents the response when the controller parameters are set at its nominal values. The dotted lines show the responses when there is $500 \%$ gain change, and the dashed lines represent the responses with $1000 \%$ gain change. From the experimental bode plots, one see that the cutting force does not have a significant effect on the positioning accuracy. Basically, the closed-loop responses maintained at a level of $10^{-3}$ to $10^{-4}$ all the way up to around $200 \mathrm{rad} / \mathrm{s}$. Probably the model-based compensator resulted in more significant response
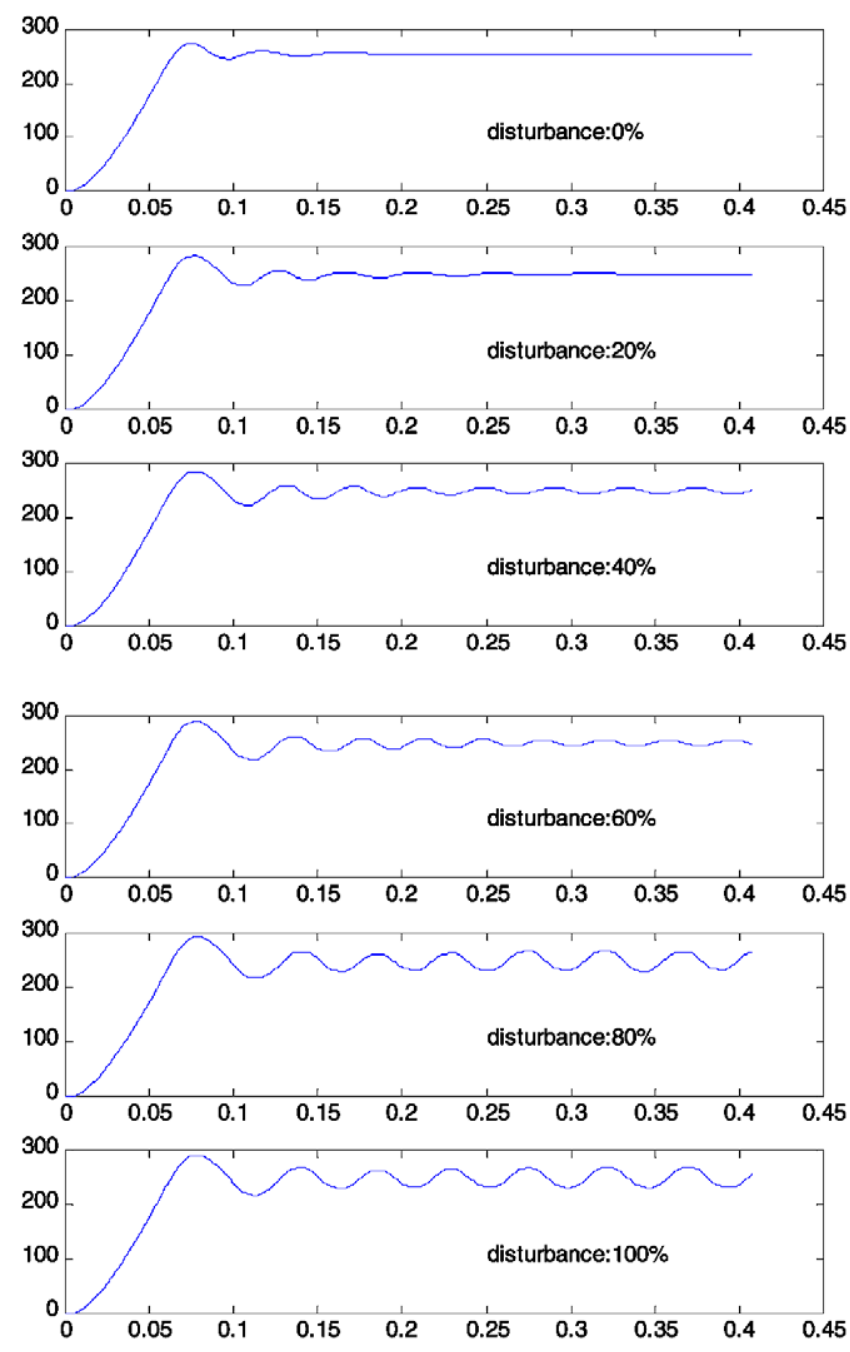

Fig. 8. Experimental result of the parameter variation effect on the PID controller. 

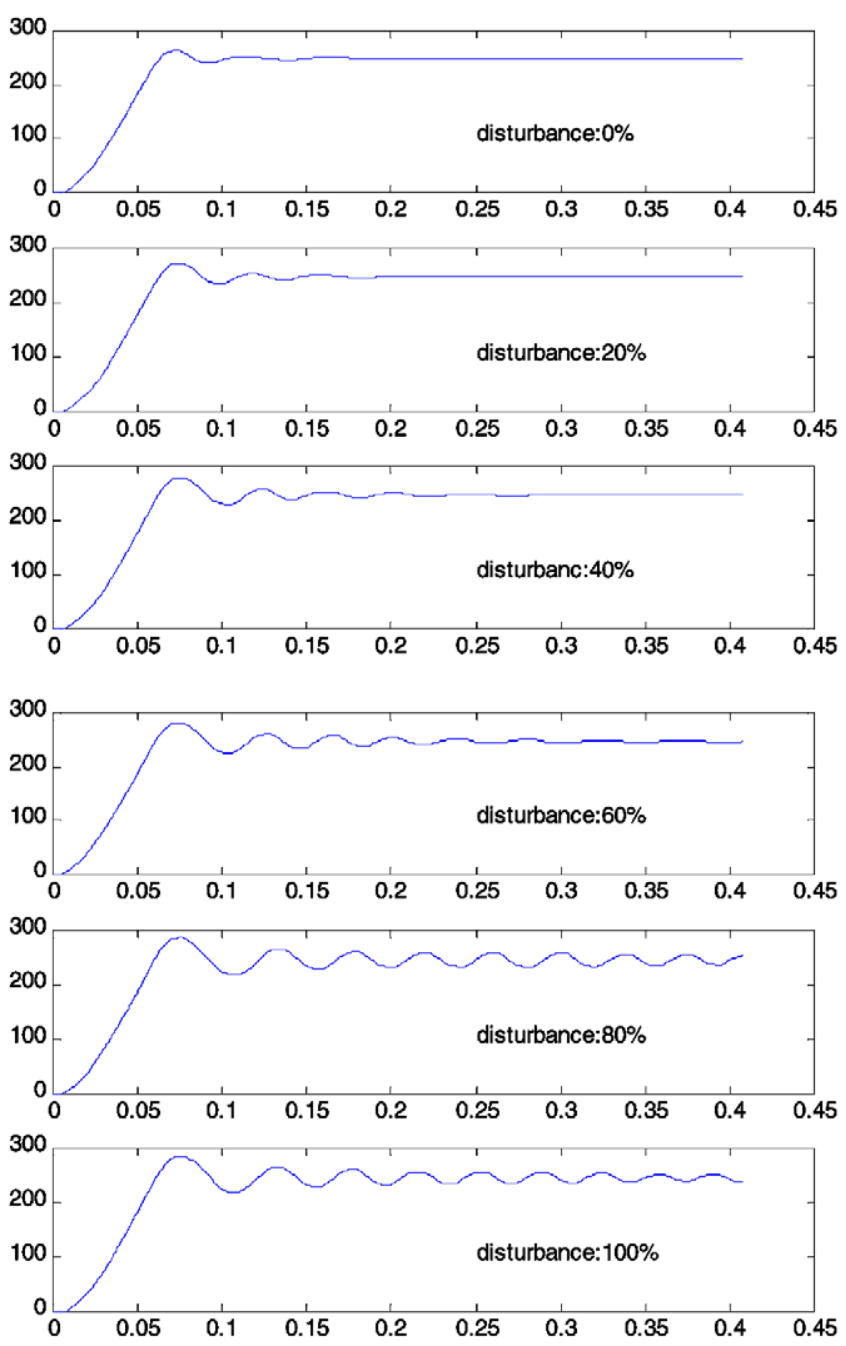

Fig. 9. Experimental result of the parameter variation effect on the PDF controller.

change. The changes in the other two control systems are not obvious. These results again matched the calculation results. The PDF controller offers the best robustness properties among the three most popular control algorithms. The experimental results are less obvious, but they do show an identical trend as in the calculation.

\section{Conclusions}

The paper investigated some of the rationales behind machine tool servo design. The basic idea is to try to analyze the effect of different servo designs and to experimentally verify the results. For this purpose, a PC-based controller on a commercial EDM machine was set up. The PC-based controller is configured so that the different types of control can be implemented. The performance robustness and the stiffness robustness against controller parameter variations were ana-
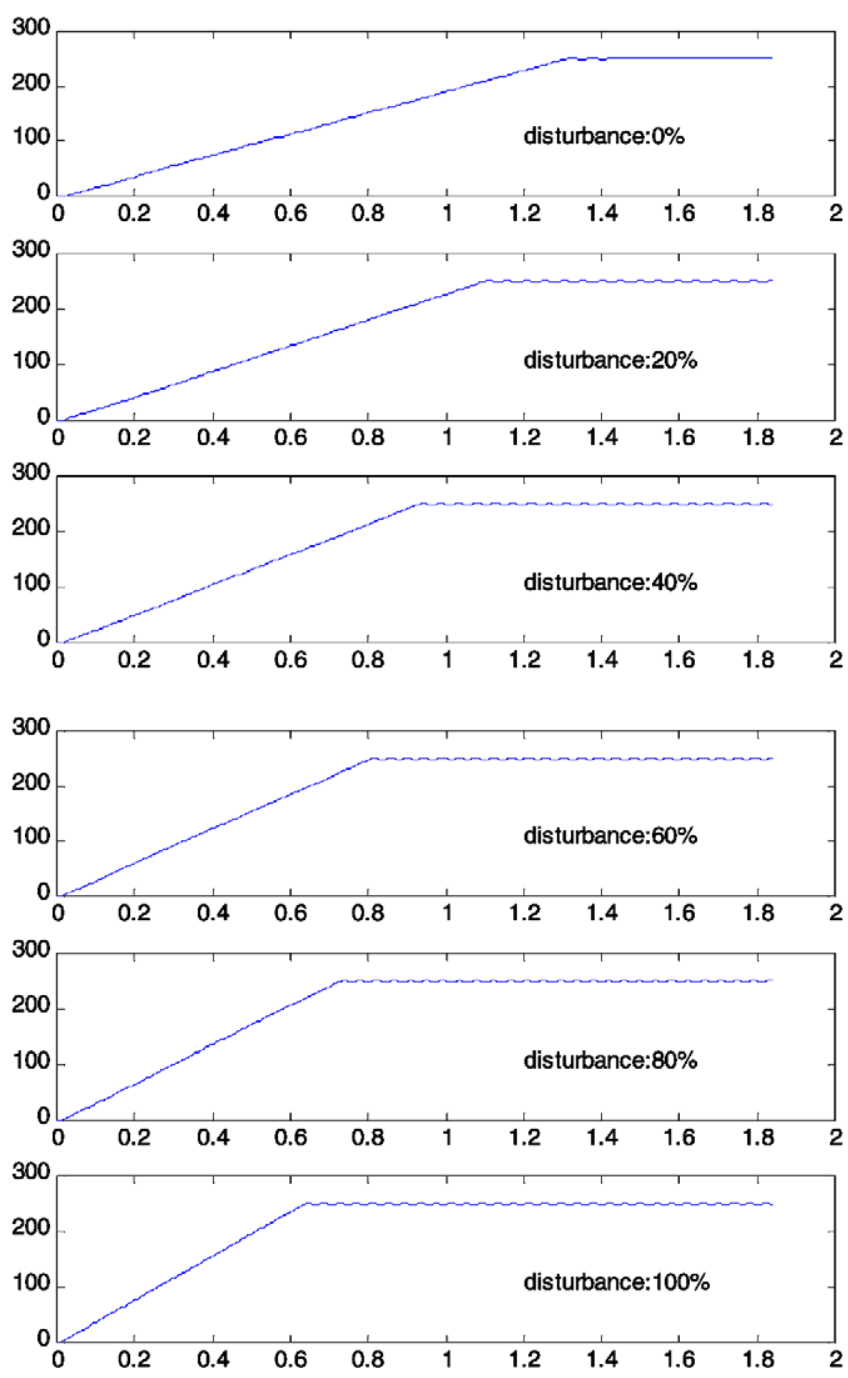

Fig. 10. Experimental result of the parameter variation effect on the model-based compensator.
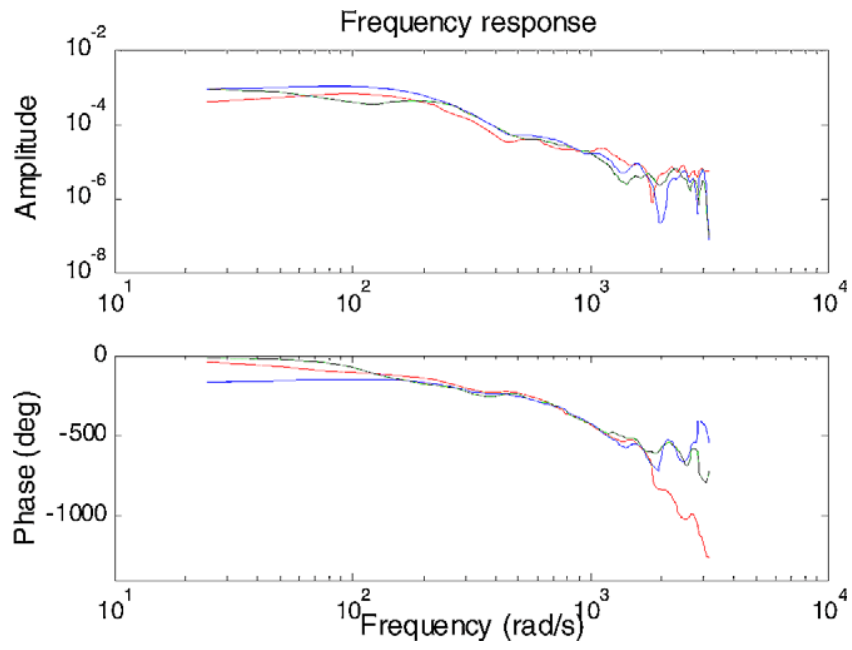

Fig. 11. Closed-loop response for the PID controller, $K_{p}=80, K_{i}=1, K_{d}=96$. 

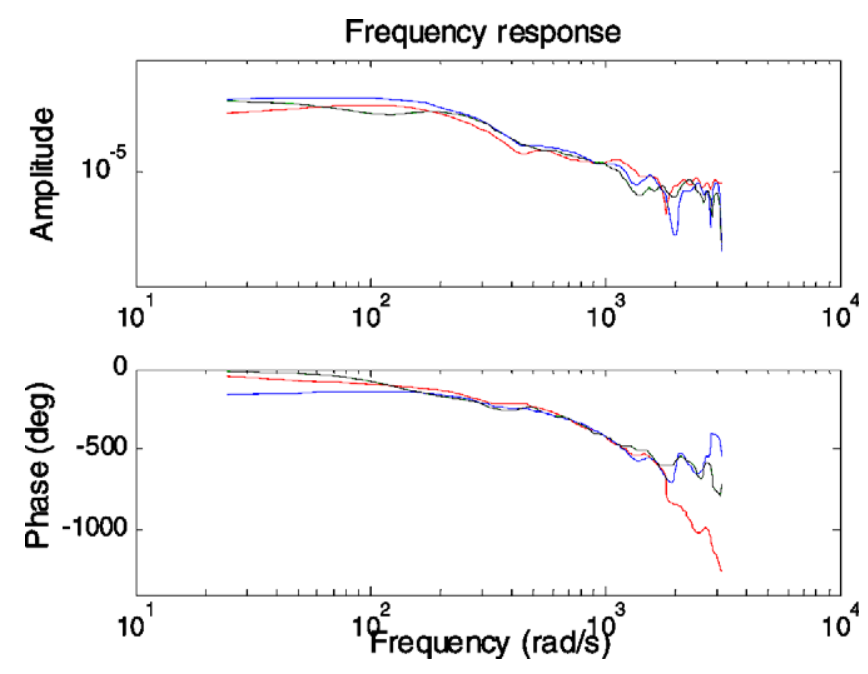

Fig. 12. Closed-loop response for the PDF controller, $K_{p}=80$, $K_{i}=8, K_{d}=80, K_{f d}=10$.

lyzed. The results from the analysis were then verified by implementing the controllers on the EDM machine. It was found that even though the traditional PID controller seems to achieve an equally good performance, the PDF controller does exhibit more robust properties against parameter variations. The model-based compensator also attains fairly good servo performance; however, it is very sensitive to any change in its gains with some form of loop recovery. Somewhat to the authors' surprise, the PDF controller exhibits far better stiffness robustness characteristics than the other two controllers. The modern MBC, on the other hand, performed poorly as fas as stiffness robustness is concerned. These results may also explain the reason that PDF controllers have been widely accepted as the core
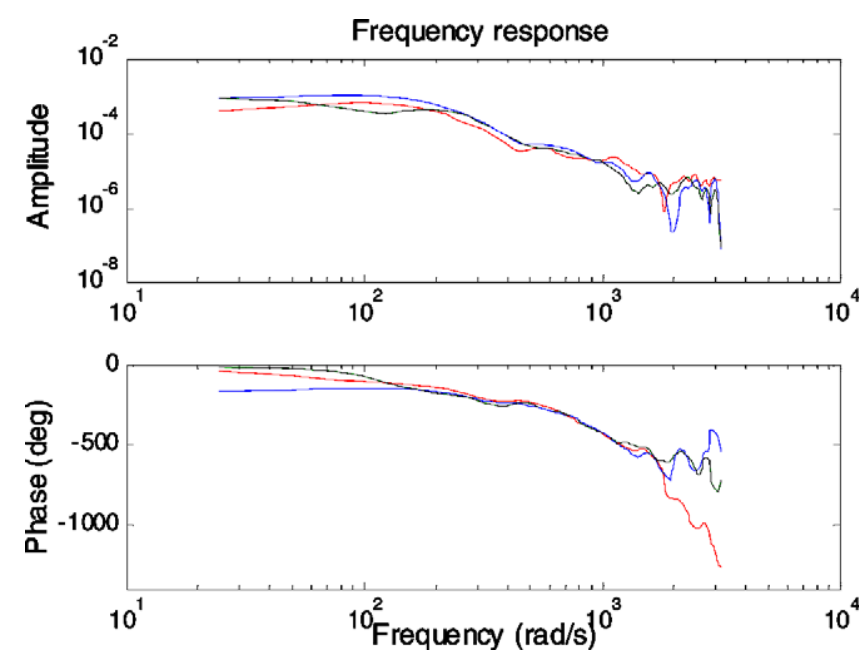

Fig. 13. Closed-loop response for MBC, $K_{M}=\left[\begin{array}{ll}0.7549 & 0.0039\end{array}\right]$, $L_{M}=\left[\begin{array}{l}203524 \\ 132542\end{array}\right]$. controller in most commercial machine tool servo controller.

\section{Acknowledgements}

This project is supported in part by the Industrial Technology Research Institute, ROC under project number $893 \mathrm{~K} 51 \mathrm{AQ} 2$, which is a subcontract from the Ministry of Economic Affairs, ROC, and in part by the National Science Council under project number NSC 89-TPC-7-002-008.

\section{References}

[1] G. Younkin, Modeling machine tool feed servo drives using simulation techniques to predict performance, Conference Record of the Industry Applications Society Annual Meeting, vol. 2, 1989, pp. 1699-1706.

[2] D.M. Alter, T. Tsu-Chin, Dynamic stiffness enhancement of direct linear motor feed drives for machining, Proceedings of the 1994 American Control Conference, vol. 3, 1994, pp. 3303-3307.

[3] B.K. Choi, C.H. Choi, H. Lim, Model-based disturbance attenuation for $\mathrm{CNC}$ machining centers in cutting process, IEEE/ ASME Trans. Mechatron. 4 (June, 2) (1999) 157-168.

[4] J.F. Cuttino, A.C. Miller Jr., D.E. Schinstock, Performance optimization of a fast tool servo for single-point diamond turning machines, IEEE/ASME Trans. Mechatron. 4 (June, 2) (1999) 169-179.

[5] A. Arakawa, K. Miyata, Simultaneous optimization algorithm for determining both mechanical-system and controller parameters for positioning control mechanisms, Proceedings of the 1996 4th International Workshop on Advanced Motion Control, AMC'96, Part 2 (of 2), March 18-21, 1996 v2 Tsu, Japan, 1996, pp. 625-630.

[6] B.-X. Xiao, The main control mode and fuzzy control strategy of $\mathrm{CNC}$ system for gear hobbing and grinding machine, Proceedings of the IEEE International Conference on Industrial Technology, 1996, pp. 643-646.

[7] J. Liu, K. Yamazaki, Y. Yokoyama, Dynamic gain motion control with multi-axis trajectory monitoring for machine tool systems, Proceedings of the 1998 International Workshop on Advanced Motion Control, AMC'98, 1998, pp. 316-321.

[8] R.J. Fornaro, T.A. Dow, A high-performance machine tool controller, Conference Record of the 1988 IEEE Industry Applications Society Annual Meeting, vol. 2, 1988, pp. 1429-1439.

[9] P. Wang, D.P. Kwok, Optimal design of pid process controllers based on genetic algorithms, Control Engineering Practice 2 (August, 4) (1994) 641-648.

[10] C.-J. Wu, C.-H. Huang, A hybrid method for parameter tuning of PID controllers, Journal of the Franklin Institute 334 (July, 4) (1997) 547-562.

[11] S.-C. Lin, Y.-Y. Chen, Design of self-learning fuzzy sliding mode controllers based on genetic algorithms, Fuzzy Sets and Systems 86 (2) (1997) 139-153, March 1.

[12] H. Ishigami, Y. Hasegawa, T. Fukuda, T. Shibata, Automatic generation of hierarchical structure of fuzzy inference by genetic algorithm, Proceedings of the 1994 IEEE International Conference on Neural Networks, Part 3 (of 7) June 27-29, 1994, vol. 3, Orlando, FL, USA, 1994, pp. 1566-1570.

[13] L. Wang, J. Yen, Extracting fuzzy rules for system modeling using a hybrid of genetic algorithms and Kalman filter, Fuzzy Sets and Systems 101 (3) (1999) 353-362, February 1. 
[14] A. Tesar, M. Drzik, Genetic algorithms for dynamic tuning of structures, Computers and Structures 57 (2) (1995) 287-295, October 17.

[15] S.V. Ulyanov, K. Yamafuji, K. Miyagawa, T. Tanaka, T. Fukuda, Intelligent fuzzy motion control of mobile robot for service use, Proceedings of the 1995 IEEE/RSJ International Conference on Intelligent Robots and Systems, Part 3 (of 3) August 5-9, 1995, vol. 3 Pittsburgh, PA, USA, 1995, pp. 486489.

[16] E.W. McGookin, D.J. Murray-Smith, Y. Li, T.I. Fossen, Ship steering control system optimisation using genetic algorithms, Control Engineering Practice 8 (April, 4) (2000) 429-443.

[17] T.S. Wu, J.C. Liu, Fuzzy control of rider-motorcycle system using genetic algorithm and auto-tuning, Mechatronics 5 (June, 4) (1995) 441-455.

[18] Y.S. Tarng, H.Y. Chuang, W.T. Hsu, Intelligent cross-coupled fuzzy feedrate controller design for CNC machine tools based on genetic algorithms, International Journal of Machine Tools and Manufacture 39 (October, 10) (1999) 1673-1692.

[19] D.Y. Ohm, Analysis of PID and PDF compensators for motion control systems, IEEE (1994).

[20] Z. Nagy, A. Bradshaw, Comparison of PI and PDF controls of a manipulator arm, IEE (1998).

[21] H. Koichi, K. Tsutomu, K. Masashi, K. Hidenori, Model-based robust control of a manipulator, IEEE (1991).

[22] F. Oliver, P. Qi, J. Gawthrop, N.R.L. Maccallum, Model-based observer: a gas turbine engine case study, IEEE (1992).

[23] TMS320F/C24x DSP Controllers CPU and Instruction Set Reference Guide, 1999.

[24] TMS320F243/F241/C242 DSP Controllers System and Peripherals Reference Guide, 2000.

[25] TMS320C1x/C2x/C2xx/C5x Assembly Language Tools User's Guide, 1995. 